\title{
Aplicación de un Modelo de Ingeniería Estratégica a una MiPyME de las Artes Gráficas: diagnóstico y estrategias.
}

Juan Víctor Bernal, 'María Antonieta Corderoii y Mireya Berenice Monroyiii

\section{Resumen}

La micro, mediana y pequeña empresa (MiPyME) es un tipo de organización que está siendo atacada por los avances tecnológicos, la competencia, los nuevos productos, y puesta en riesgo por sus problemas de dirección. Muchas adolecen de no tener una visión que guie su actuar, lo que provoca que sus esfuerzos se vean acotados estrechamente por problemas que, si no se superan, terminan sepultándola. La industria de las artes gráficas se ha visto golpeada por la invasión de dichos avances tecnológicos, lo que la pone en una época muy importante de renovación, como nunca antes en la historia. Este trabajo presenta un análisis de una MiPyME dedicada a la fabricación de equipo para serigrafía que, como toda industria, enfrenta los embates de esta situación, por lo que se realiza un estudio que tiene como resultado la generación de un diagnóstico de su actual situación, para proponer estrategias que permitan su sobrevivencia y el éxito futuro, en mercados cambiantes y emprendedores.

Palabras clave: MiPyME, diagnóstico, estrategia, SWOT, análisis.

Tecnológico de Estudios Superiores de Cuautitlán Izcalli, México. Profesor de la carrera de Ingeniería en Logística y Líder de Línea de Investigación del Cuerpo Académico Desarrollo Tecnológico.

ii Tecnológico de Estudios Superiores de Cuautitlán Izcalli, México. Profesor de la carrera de Ingeniería en Gestión Empresarial y Secretaria del Cuerpo Académico Desarrollo Tecnológico.

iii Tecnológico de Estudios Superiores de Cuautitlán Izcalli, México. Profesor de Tiempo completo de la carrera de Ingeniería en Administración y Líder del Cuerpo Académico Desarrollo Tecnológico. 


\section{Abstract}

The micro, small and medium business (MSMB) is a kind of organization that are being attacked by technological advances, competitors, new products, and it is putting on risk by their management problems. Many of these suffer from not having a vision to guide their actions, which causes their efforts be closely bounded by problems that, if unaddressed, they end up burying. The graphic arts industry has been touch by the invasion of these technological advances, which puts it in a very important time of renewal, as never before in history. This study presents an analysis of MSMB engaged in the manufacture of equipment for screen printing process, as this whole industry, faces the brunt of this situation, so a study that results in the generation of a diagnosis of the current situation, is made to propose strategies to their survival and success in a very changing and turbulent markets.

Key words: Small business, diagnosis, strategy, SWOT, analysis. 


\section{Introducción}

Cierto es que gran parte de la economía de una nación se sustenta en la micro, mediana y pequeña empresa (MiPyME), pues en número y potencialidad, es un sector estratégico pujante y que, al paso de los años, se ha convertido en un detonador importante para generación de empleos y dinamismo en el desarrollo económico de un país.

Una característica elemental de la MiPyME es su limitado nivel de recursos, que le da muy poco margen de error. La racionalidad y maximización de beneficios es parte de la misión de una empresa (Canals, 2008). No hay colchón para largos aprendizajes ni para defenderse de los grandes errores de cálculo o de las sorpresas o de las complacencias productivas. (Resnik, 1992). Es vital calcular cada paso que se debe dar.

En Latinoamérica, es un componente importante en la cantidad de las empresas que dinamizan la economía. Por ejemplo, México tiene cerca del 95\% del número que conforma la economía nacional (INEGI, 2011), y Argentina es del 98\%, según la Subsecretaría de Desarrollo de Inversiones y Promoción Comercial de Argentina (2015), y dentro de los sectores en que se clasifica la economía, el secundario, siendo en la transformación, las Artes Gráficas una de las ramas más golpeadas. La Unión Industrial Argentina, en su último informe (2016), menciona una caída en sus ingresos en los últimos cuatro años, reportando un decremento del $14.1 \%$ en papel, cartón, imprenta y publicaciones, en relación con su principal socio comercial, Brasil.

En México, según el presidente de la Cámara Nacional de la Industria de las Artes Gráficas, los avances tecnológicos en los sistemas de impresión por plotters y el crecimiento de los servicios digitales de propaganda y facturación, por citar algunos ejemplos, ha derivado en el cierre de entre mil y dos mil imprentas en 2013 y 2014 (CANAGRAF, 2015).

En forma particular, la industria de la fabricación de equipo para serigrafía, ha visto detenido su crecimiento por varios factores, entre ellos la nula generación de esquemas que le permitan una adecuación y un crecimiento entre las cambiantes condiciones del mercado y de la competencia.

Es imperativa la implementación de estrategias que permitan mejorar los procesos internos y externos de la organización, integrando innovaciones, consecuencia de incrementar los niveles de capacitación; sólo así será posible aumentar su productividad y mejorar las condiciones laborales de los trabajadores.

El uso de la tecnología en el sector de las artes gráficas, enfrenta un entorno cambiante, resultado de la apertura comercial y el impacto para adquirir y negociar tecnologías, a falta de desarrollo de aquellas propias, por tal motivo es imperante la necesidad de diseñar un modelo de ingeniería estratégica capaz de generar nuevos procesos, productos o servicios. 
A pesar de que el sector de la MiPyME en México, constituye el 99\% del tejido industrial y que generan la mayoría de los empleos, su nivel de productividad es extremadamente bajo en relación con las grandes empresas (CEPAL, 2014). Esta situación es el reflejo de la poca cobertura de mercados nacionales y la poca motivación para la internacionalización, careciendo de una visión exterior que identifique las necesidades del sector serigráfico para desarrollar tecnología que impacte en los mercados externos.

La internacionalización se complica en el sector serigráfico al no contar con suficiente información para exportar, infraestructura limitada, altos costos de transporte y burocratización en los tramites, así como un reducido acceso a programas de financiamiento (Amarante, 2016).

Las revoluciones empiezan mucho antes de que se declaren oficialmente. Durante varios años, los ejecutivos de alto nivel en una amplia gama de industrias han replanteando la forma de medir el rendimiento de sus negocios. Han reconocido que las nuevas estrategias y realidades competitivas demandan nuevos sistemas de medición. Ahora éstos están profundamente involucrados en la definición y el desarrollo de esos sistemas para sus empresas (Eisner, 2006).

Trazar el mejor camino para una organización no es una tarea fácil. A veces se cree que es suficiente un ligero diagnóstico, en otras, que el realizar pesados análisis puede no ser suficientes para determinar el rumbo. Reinventar estrategias es la meta de cada día para muchas empresas que no terminan por identificar hacia dónde van; sin embargo, eso no demerita su esfuerzo a la hora de emprender este nuevo camino como un reto mayor.

Para aplicar un Modelo de Ingeniería Estratégica, se necesita partir de los resultados de un diagnóstico, usando técnicas de análisis gráficos de mediciones empresariales como ingresos, egresos, y utilidad, complementándolo con el análisis SWOT, y el estudio de las leyes de fuerza del mercado. Una vez hecho esto, se deben establecer estrategias, mismas que se deben alinear con las acciones de la empresa, usando el despliegue en cascada (Hoshin Kanri), que midan el desempeño a través de indicadores, empleando el concepto de Balanced Scorecard, para cuantificar el cumplimiento de metas y objetivos.

Por consiguiente, este proyecto busca exponer la primera parte de ese modelo, a través del diagnóstico con la información disponible, para finalizar con la definición de la estrategia empresarial más adecuada que sea motivo de éxito en la MiPyME, en el contexto actual, que sea la base para lograr una aplicación más general a diferentes industrias y tamaños de organización. 


\section{Marco teórico}

\section{Análisis SWOT}

Se emplea para ponderar las capacidades y deficiencias de los recursos de una empresa, sus oportunidades comerciales y las amenazas externas de su bienestar futuro (Thompson, Strickland \& Gamble, 2015). Sirve de guía para determinar cómo se encuentra la empresa en este momento, es una herramienta administrativa para analizar y evaluar una organización y para saber cómo se encuentra en el entorno en donde se desenvuelve. Debe enfocarse solamente hacia los factores claves para el éxito de la organización.

Así que, fundamentado en lo anterior, el análisis SWOT es de gran ayuda para determinar el diagnóstico actual de la empresa. Las debilidades y las fortalezas son internas a la organización, mientras que las oportunidades y las amenazas son externas. Mientras que las fortalezas y las oportunidades son positivas para la organización, las debilidades y amenazas no lo son (Santiago, 2006).

Existen muchos factores externos que inciden en el desarrollo de una organización, por ello es muy importante que las organizaciones estudien las fuerzas políticas, legales, tecnológicas, sociales y macroeconómicas, porque ellas impactarán a la planeación y sus resultados (Hill \& Jones, 2011). En conjunto, las fortalezas, oportunidades, debilidades y amenazas debieran ser tratadas como un sistema (Hermida, 1992). Aunque desde luego que todos en la organización son responsables de identificar los elementos del SWOT (Corona, 1998).

Es muy importante considerar que tiene sus limitaciones, ya que es sólo un punto inicial del análisis empresarial. Al haber hecho el SWOT se tiene la materia prima para estructurar un análisis estratégico con mayor profundidad. No se debe considerar como un fin, sino como un medio (Eisner, 2006).

\section{Modelo de las cinco Fuerzas}

Se emplea para diagnosticar de manera sistemática las principales presiones competitivas de un mercado y para evaluar la fortaleza e importancia de cada una (Thompson, Strickland \& Gamble, 2015). Las cinco fuerzas influyen en los precios, costos y requisitos de inversión, que son los factores básicos que determinan la rentabilidad. El modelo, creado por Michael E. Porter (1980), establece los protagonistas (competidores, compradores, proveedores, posibles nuevos competidores y sustitutos) y sus interrelaciones (las cinco fuerzas) con los factores que determinan la intensidad de dichas fuerzas. 


\section{La Estrategia}

Es un patrón, establece una dirección, concentra el esfuerzo, define a la organización y proporciona consistencia. Una buena estrategia permitirá alcanzar los objetivos de la organización utilizando los recursos disponibles y una mala conducirá a la ruina, a problemas para la organización o a no alcanzar los objetivos que se plantearon (Mintzberg, 2008).

Existen cinco estrategias competitivas genéricas, desarrolladas por Michael E. Porter: de bajos costos, de diferenciación amplia, del costo más bajo, centrada (o de nicho de mercado) basada en costos bajos, y centrada basada en la diferenciación. Cada uno de estos posicionamientos genéricos persigue una posición distinta en el mercado, e implica enfoques diferentes para competir y operar en el negocio (Thompson, Strickland \& Gamble, 2015).

\section{Marco contextual}

\section{La empresa}

Dentro de los sectores en que se clasifica la economía, el secundario, relativo a la transformación, una de las ramas más golpeadas es la de artes gráficas, en particular la industria de la fabricación de equipo para serigrafía, que, en México, se ha visto detenido su crecimientos por varios factores, entre ellos, los avances tecnológicos en los sistemas de impresión por plotters y el crecimiento de los servicios digitales de propaganda y facturación, por citar dos ejemplos, y que no han podido hacer frente.

La empresa que es objeto de estudio se encuentra localizada en el Estado de México, en el límite norponiente de la Ciudad de México. Se dedica a la impresión de equipo y maquinaria para la impresión por serigrafía, considerando equipos de pre prensa, como los marcos de vacío, para la exposición a la luz de marcos serigráficos o sablones, y tinas de revelado [ver figura 1]; equipos de secado o curado, como los hornos de luz ultra violeta [ver figura 2]; y de impresión, que es el producto líder, un equipo de impresión automática que se muestra en la figura 3. 

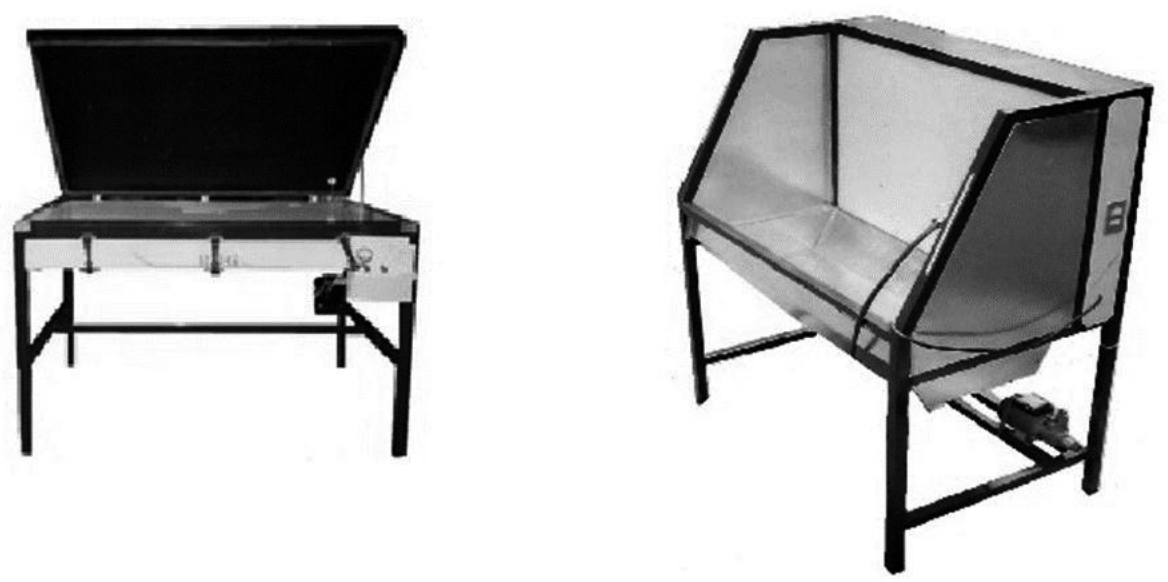

Fig. 1 . Equipos para pre prensa que fabrica la empresa. A la izquierda, el marco de vacío, a la derecha, tina de lavado para revelado de sablones o marcos serigráficos.

Fuente: propia.

Es conveniente mencionar que la serigrafía es un procedimiento de impresión que consiste en el paso de la tinta a través de una malla de poliéster sobre un bastidor. Se hace pasar aplicando presión con un rasero o racleta con punta fabricada por elastómero.

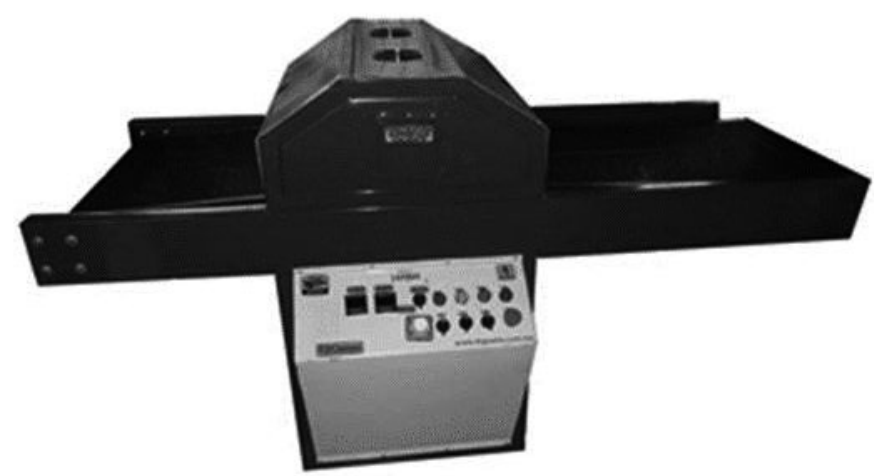

Fig. 2. Horno de secado y curado para tintas ultra violeta o UV. Fuente: Propia.

La malla tiene tejidos abiertos y bloqueados por una emulsión, normalmente hecha por una mezcla de alcohol de polivinilo con una solución que le da la propiedad de ser sensible a la luz. Esto produce un efecto enmascarante que define la figura que se desea imprimir. La impresión se puede hacer de forma manual o a través de equipos automáticos que incrementan los niveles de productividad de los impresores. 


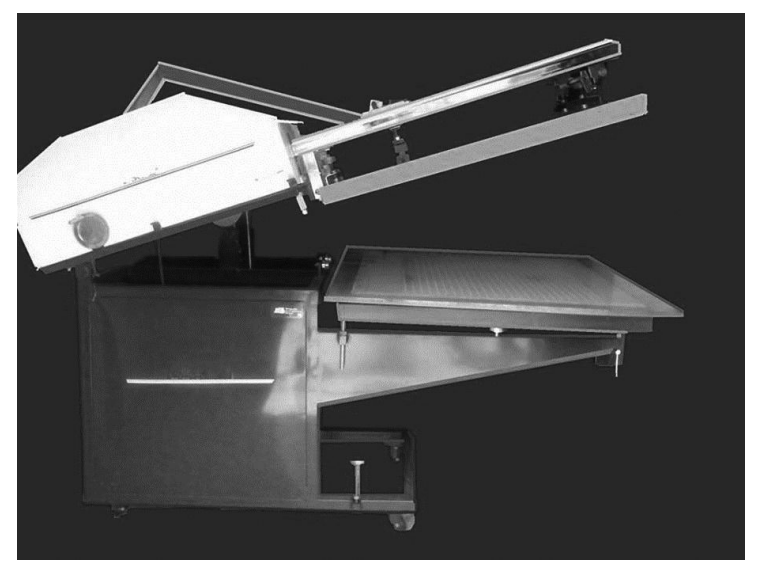

Fig. 3 . Equipo de impresión automática por serigrafía en plano. Fuente: Propia.

\section{Desarrollo}

\section{Metodología}

Para el desarrollo de este trabajo se utiliza una metodología hipotética experimental, que consiste en generar una hipótesis nula que será probada a través de un modelo de pronósticos ajustado por medio de una técnica de regresión lineal, con el propósito de rechazar o no rechazar su validez. Dicho modelo se crea a partir de los datos históricos obtenidos en el tiempo de estudio. Con esto, se genera un análisis SWOT que permite proponer las estrategias necesarias para que la empresa pueda tener mayores posibilidades de éxito en el futuro inmediato.

\section{Hipótesis Nula}

La hipótesis nula que se formula es la siguiente: se considera que la empresa sigue una estrategia actual definida que se debe observar reflejada al paso del tiempo, sobre el promedio de ventas mensuales. Esto permitirá concluir una mejora en el nivel de ventas a futuro, y que dicha estrategia actual es la adecuada.

\section{Análisis de los datos}

Se realizó un estudio de tendencia de las ventas efectuadas en los últimos 30 meses de operación. Por petición de la empresa, se omiten valores puntuales, sin embargo, se muestran datos finales tomados del procesamiento para esta publicación [ver figura 4]. 


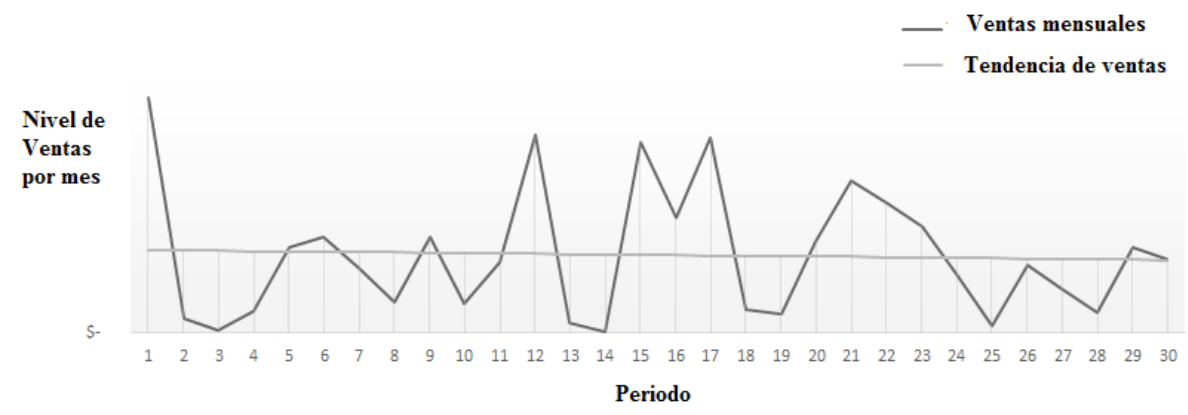

Fig. 4 . Nivel y tendencia de ventas de la empresa en los últimos 30 meses.

Fuente: Propia.

Se puede apreciar que hay una gran dispersión en los resultados de las ventas, ya que en algunos periodos se disparan y en otros el monto es casi cero. Ocupando la herramienta de regresión lineal de una hoja electrónica por computadora [ver tabla 1], se consigue un modelo que tiene una razón de cambio negativa, lo que está provocando que cada vez el importe por las enajenaciones sea menor, según se observa en la pendiente de la ecuación (1).

$$
Y=-15.56 X+3802.22
$$

Donde $\mathrm{Y}$ es el valor, en dólares, de las ventas por periodo; $\mathrm{X}$, el periodo del mes que se proyecta.

\begin{tabular}{|c|c|c|c|c|}
\hline Resumen & & & & \\
\hline Estadisticas de la reg & sión & & & \\
\hline Coeficiente de correlación múltiple & 0.046316476 & & & \\
\hline Coeficiente de determinación $\mathrm{R}^{\wedge} 2$ & 0.002145216 & & & \\
\hline $\mathrm{R}^{\wedge} 2$ ajustado & -0.033492455 & & & \\
\hline Error típico & 3007.237361 & & & \\
\hline Observaciones & 30 & & & \\
\hline ANÁLISIS DE VARIANZA & & & & \\
\hline & Grados de libertad & Suma de cuadrados & Promedio de los cuadrados & \\
\hline Regresión & 1 & 544373.6839 & 544373.6839 & \\
\hline Residuos & 28 & 253217343.2 & 9043476.544 & \\
\hline Total & 29 & 253761716.9 & & \\
\hline & Coeficientes & Error tipico & Estadistico $t$ & Probabilidad \\
\hline Intercepción & 3802.227735 & 1126.128623 & 3.376370742 & 0.00217074 \\
\hline Pendiente & -15.56319328 & 63.43338073 & -0.245347057 & 0.8079771 \\
\hline
\end{tabular}

Tabla 1. Datos parciales de la regresión lineal aplicada a los datos de ventas de la empresa.

Fuente: Propia. 
La consecuencia de este comportamiento es que, en un futuro próximo, la compañía tendrá menos ingresos que reducirán sus niveles de liquidez, pero si llegan a ser menores que sus gastos fijos, ya no será viable la operación.

\section{Comprobación de la hipótesis}

Montgomery \& Runger (2013) proponen una metodología de 8 pasos que permite concluir la validez de la hipótesis nula. En este caso, se usa una prueba para la significancia de la regresión lineal, usando un nivel $\alpha=0.01$, una pendiente estimada $\hat{\beta}_{1}=-15.56$, con una varianza estimada $\hat{\sigma}^{2}=9043476.544$. El desarrollo de dicha prueba se muestra en la figura 5 .

1. Prueba de significación de la regresión lineal.

2. Hipótesis nula. $H o: \beta_{1}=0$

3. Hipótesis alternativa. $H a: \beta_{1} \neq 0$

4. Nivel de significancia: $\alpha=0.01$

5. Estadístico de prueba. $T_{0}=\frac{\widehat{\beta}_{1}-\beta_{1,0}}{\sqrt{\frac{\hat{\sigma}^{2}}{S_{x x}}}}$

6. Criterio de rechazo. Rechazar Ho si $T_{0} \geq 2.7633$

7. Cálculos. $T_{0}=\frac{-15.56-0}{\sqrt{\frac{9043476.544}{2247.5}}}=-0.2453$

8. Conclusión. No se rechaza $\boldsymbol{H o}$, por lo que no hay una relación lineal $x-y$.

Fig. 5 .Desarrollo del procedimiento de la prueba de hipótesis para la empresa. Fuente: Propia.

Con esto, la conclusión es contundente: los datos de las ventas mensuales no siguen un patrón definido por una estrategia planeada, es más, se puede apreciar que no existe, moviéndose los resultados por cuestiones puramente aleatorias.

\section{Análisis SWOT}

Los resultados del análisis SWOT se muestran en la tabla 2. Observando sus cuatro ventanas, se puede hacer el siguiente diagnóstico: la empresa se encuentra en un estado 
de detención, pero todavía sana, ya que no tiene amenazas a corto y mediano plazo como deudas, demandas o huelgas. Sin embargo, a largo plazo, la entrada de nuevas tecnologías la pueden volver vulnerable si no desarrolla innovaciones en productos, procesos y servicios que la mantengan a niveles competitivos.

Para esto, sus fortalezas le pueden soportar generar dichas innovaciones. Sus debilidades se pueden corregir a través de planes y programas que le ayude a crear capital humano. En términos generales, aunque débil, la empresa es viable para crecer y crear utilidades.

\begin{tabular}{|c|c|}
\hline Fortalezas & Debilidades \\
\hline $\begin{array}{l}\text {-Flexibilidad en los procesos de producción. } \\
\text {-Experiencia reconocida en el mercado. } \\
\text {-Trayectoria con más de } 30 \text { años. } \\
\text {-Se conocen los procesos de producción. } \\
\text {-Proveedores sólidos y con una buena relación comercial. } \\
\text {-Clientes importantes con más de } 15 \text { años atendiéndolos. } \\
\text {-No hay deuda importante en el negocio. }\end{array}$ & $\begin{array}{l}\text {-No hay un orden en las operaciones de la cadena de } \\
\text { suministro ni en producción. } \\
\text {-Capital financiero es limitado. } \\
\text {-La dirección no tiene una mayor visión a futuro. } \\
\text {-Su estructura jerárquica es pobre. } \\
\text {-No tiene procesos documentados. } \\
\text {-No hay actualizaciones tecnológicas en procesos. } \\
\text {-Las actualizaciones tecnológicas son escasas en el portafolio } \\
\text { de productos. } \\
\text {-No hay campañas de publicidad que generen ventas. } \\
\text {-No hay una estructura definida para calcular costos de } \\
\text { producción. } \\
\text { - Falta de capacitación sobre aspectos de producción, } \\
\text { administrativos y tecnológicos. }\end{array}$ \\
\hline
\end{tabular}

Tabla 2. Resultados del análisis SWOT aplicado a la empresa.

\section{Diagnóstico}

La empresa tiene una tendencia de ventas con una pendiente negativa. Cada vez vende menos pero no lo han apreciado sus directivos. No presenta grandes pasivos, lo que le da fortaleza. Tiene un buen producto que se mueve en el mercado de forma aleatoria. Su proceso de producción y cadena de suministro no es eficiente, hay fallas. El personal no está del todo capacitado para atender las órdenes de trabajo que se presentan. Existen amenazas que surgen de las nuevas tecnologías en productos sustitutos.

Ha construido a la fecha una posición en el mercado y experiencia en el ramo. Tiene un gran potencial para que sea una entidad generadora de ingresos y fuente de empleos, pero hay que desarrollar a la empresa. 


\section{Desarrollo de la estrategia}

Debe seguir la estrategia de fortificarse y defenderse, usando variaciones de su estrategia actual, y luchar por mantener sus ventas, participación en el mercado, rentabilidad, y posición competitiva en el nivel en que se encuentra (Thompson, Strickland \& Gamble, 2015). La actual estrategia ha generado un débil desempeño, por lo que, para remodelarla hay que seguir varios caminos:

a) Cambiarse a un nuevo enfoque competitivo para reestablecer la posición de la empresa en el mercado.

b) Revisar las operaciones internas y las estrategias de las funciones para sostener mejor la misma estrategia comercial general.

c) Hacer alianzas con empresas del sector para mejorar su desempeño.

d) Centrarse en el núcleo reducido de productos y clientes que concuerde con sus fortalezas.

e) Usar publicidad impresa y en redes sociales, enfocados a llegar exclusivamente a empresas del ramo de las artes gráficas.

Para fortalecer sus ingresos debe incrementar el volumen de ventas. Las opciones principales para acumular ingresos son rebajas, mayor publicidad, mayor atención de ventas, agregar servicios al cliente y hacer mejoras al producto rápidamente.

Es importante observar una mayor eficiencia operativa que permita reducir costos, por lo que es necesario que se puedan hacer arreglos en la cadena de suministros interna, usando la filosofía lean, de forma tal que solo se contemplen las operaciones que le agreguen valor al producto, a través de una secuencia que permita hacer fluir las operaciones de manufactura con un orden lógico.

De la mano con estos esfuerzos, las personas claves de la compañía deben comenzar a capacitarse en temas de operación, manufactura, administración y ventas, que permitan aterrizar mejor la propuesta y lograr los avances requeridos. Se debe mostrar un interés por generar innovaciones en los procesos y productos, que se desprendan de esta capacitación.

\section{Resultados y conclusiones}

A pesar del poco crecimiento del sector serigráfico en América Latina en los últimos años, se puede comprender que los factores que limitan el crecimiento se orientan principalmente a la falta de un plan de desarrollo en el corto y largo plazo, que imposibilitan una buena organización e integración de la MiPyME, incluyendo la falta de dirección, y control de las operaciones del área productiva, que no la hacen competitiva.

Desde la perspectiva del ánalisis realizådo en la MiPyME, el obtener una pendiente negativa de -15.56 dólares determina que puede llegar a disminuir sus 
ganancias y rebasar el punto de equilibrio, que de continuar la operación de la empresa como hasta ahora, la consecuencia lógica será el cierre del negocio y la pérdida de fuentes de trabajo.

El SWOT indicó que el comportamiento aleatorio de las ventas de la empresa no tiene una estrategia definida, por lo que se debe definir las fuerzas del mercado que le permitan impactar positivamente en sus ingresos.

La empresa puede tener éxito si lleva a cabo el despliegue de las estrategias a través del Hoshin Kanri en tácticas y operaciones que involucren los niveles jerárquicos; un Balance Scorecard que proporcione las métricas para medir el desempeño, sin olvidar el sentido holístico que debe llevar el trabajo a futuro.

Este Modelo se puede aplicar a cualquier tipo de industria con problemas e incertidumbres en sus procesos, mejorando los resultados al incluir una experiencia que les permita comprender y dominar las situaciones complicadas, con beneficios para los grupos de interés, en los que se incluyen empleados, empresarios, gobierno, sociedad y el país que los envuelve.

\section{Referencias}

Amarante, V. (2016). Se requiere una nueva generación de políticas dirigidas a las MiPyME. Recuperado de http://www.cepal.org

Canals, J. (2008). En busca del equilibrio. Consejos de Administración y alta dirección en el gobierno de la empresa. España: Prentice Hall.

Corona, R. (1998). Estrategia. México: Ediciones SICCO.

CANAGRAF (2015). Actividades del Presidente. Recuperado de: http://canagraf. $\mathrm{mx} /$ index.php/actividades-del-presidente.html

CEPAL (2014). Taller con empresas e instituciones sobre innovación para exportar al mercado de EE UU. División Comercio Internacional e Integración. Recuperado de http://www.cepal.org

Eisner, A. B. (2006). Strategic management. Text and cases. New York, NY, Estados Unidos: Mc Graw Hill / Irwin.

Hermida, J. (1992). Administración \& Estrategia. Buenos Aires, Argentina: Ediciones Macchi.

Hill, C. W. \& Jones, G. R. (2011). Administración estratégica, un enfoque integrado. México, D. F.: México. Progreso, S. A. de C. V. 2011.

Mintzberg, H. (2008). Safari a la Estrategia. México: Ediciones Granica, S. A, 
Montgomery, D. \& Runger, G. (2013). Probabilidad y Estadística aplicadas a la ingeniería. México: Editorial Limusa S.A. de C. V.

Porter, M. (1980). Competitive Strategy: Techniques for Analyzing Industries and Competitors. New York: Free Press,

Santiago, G. D. (2006). Dirección estratégica. Madrid: Mc Graw Hill Interamericana de España, S. A.

Thompson, A., Strickland, A. \& Gamble, J. (2015). Administración estratégica. Teoría y casos. México: Mc Graw-Hill.

Resnik, P. (1992). Como dirigir una pequeña empresa. Decálogo del éxito y la supervivencia. México: Mc Graw- Hill.

Subsecretaría de Desarrollo de Inversiones y Promoción Comercial, (2015). Empresas en Argentina. Guía del Inversor. Invierta en Argentina. Recuperado de http://inversiones.gob.ar/es/economia-y-negocios

Unión Industrial Argentina (2016). Análisis de la situación con Brasil y potenciales riesgos de corto plazo en el empleo. Recuperado de https:/gallery.mailchimp. com/cbf0b6d614f593e54a4cf761b/files/Informe_Situaci\%C3\%B3n_con_ Brasil.pdf 